\title{
Migración de retorno de adultos mayores a México: redes sociales, familia y acumulación
}

\author{
Sandra N. Martínez Díaz Covarrubias*
}

Recepción: 25 de septiembre de 2017 Aceptación: 30 de noviembre de 2017

Resumen El retorno de adultos mayores a México es un tema aún poco explorado en la literatura sobre migración. Este artículo presenta una discusión sobre algunos estudios que han abordado la situación de adultos mayores en retorno; más allá de la idea del migrante de retorno en etapa de jubilación o edad avanzada, que terminó su vida laboral en Estados Unidos y regresa a vivir su retiro con algún tipo de pensión, existe otro tipo de retorno de adultos mayores en condiciones de precariedad y austeridad, que provocan una acumulación de desventajas y dificultan la integración laboral y económica. Las redes familiares y sociales desempeñan un papel fundamental en la integración social de estas personas migrantes de retorno y también para solventar, en cierta forma, algunas de las carencias que enfrentan. Así mismo, se ofrece una reflexión sobre la falta de políticas públicas orientadas a esta población.

PALABRAS CLAVE: migración de retorno, México-Estados Unidos, adultos mayores, integración, políticas públicas, acumulación de desventajas.

\section{Return migration of older adults to Mexico: Social networks, family and the accumulation of disadvantages}

Abstract The return of older adults to Mexico is an issue that is still little explored in the literature on migration. This article presents a discussion of a number of studies that have addressed the situation of older adults on their return; beyond the idea of the return migrant who has retired or reached old age, who has ended his working life in the US and comes back to live in Mexico

Doctorante en el Centro de Investigaciones y Estudios Superiores en Antropología Social (CIESAS Occidente), sandralide@gmail.com 
with some kind of pension, there is another type of returning older adult who lives in conditions of precariousness and austerity, which generate an accumulation of disadvantages that hinder labor and economic integration. The social networks and the family play a fundamental role for the social integration of these returning migrants and also to solve some of the deficiencies they encounter. Likewise, this article offers a reflection on the lack of public policies oriented to this population.

KEY WORDS: Return migration, Mexico-USA, old age, integration, public policy, cumulative disadvantages.

\section{Introducción}

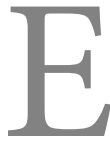

1 endurecimiento de las leyes antiinmigrantes en Estados Unidos, la crisis financiera que estalló en el año 2008 y diversas situaciones sociales y políticas en ese país en los últimos diez años han detonado un importante aumento del retorno (voluntario o no) de migrantes mexicanos de Estados Unidos a México (Massey, Pren, \& Durand, 2009; Canales, 2012; Gandini, Lozano, \& Gaspar, 2015). Se estima que durante la administración de Barack Obama (2009-2016) se registraron casi tres millones y medio de eventos de deportación de mexicanos a México. ${ }^{1}$ A este tipo de migración de retorno se le conoce como forzada, ya que se da en condiciones en que la persona es obligada a "regresar" a México. Sin embargo, existen varios otros tipos de migración de retorno que no se contabilizan tan rigurosamente como las deportaciones y, por lo tanto, no representan a todo el flujo migratorio de retorno (Giorguli, Angoa, \& Villaseñor, 2014).

El contexto actual de la migración de retorno a México es considerado por algunos autores como una nueva fase de la historia del circuito migratorio MéxicoEstados Unidos (Canales, 2012; Durand, 2013; Freidenberg \& Durand, 2016). En estos últimos diez años se estima que el volumen de la emigración de México a ese país es muy similar o incluso inferior al volumen de migración de retorno, es decir, se ha considerado como saldo neto migratorio cero (Passel, Cohn, \& González, 2012; Zenteno, 2012). Este hecho no se había presentado en otras etapas de la extensa historia migratoria entre los dos países, excepto en el periodo de las deportaciones masivas de la Gran Depresión en los años treinta del siglo pasado (Alarcón, 2015).

La Unidad de Política Migratoria (UPM), dependencia encargada de reunir la estadística sobre flujos migratorios en México, entre ellos las repatriaciones, ha registrado para el periodo 2008-2016 un total de 3,426,790 eventos de repatriación de Estados Unidos a México. Si bien una persona puede tener varios de estos eventos, la cifra de personas es un estimado del periodo 2009-2016 de acuerdo con los eventos de repatriación totales. 
Actualmente, bajo la administración de Donald Trump a partir de 2017, se ha agudizado el discurso racista y de odio hacia la población inmigrante, además de que han aumentado las disposiciones legales que permiten su detención.

Un aspecto que ha tomado relevancia académica en este cambio en el patrón migratorio entre ambos países es que ha variado la composición demográfica de los migrantes de retorno. Si bien su perfil clásico era el de un hombre en edad productiva o al final de ella retornando a su lugar de origen en alguna comunidad rural, el nuevo gran flujo de retorno es mucho más heterogéneo: compuesto por niños, niñas, adolescentes, jóvenes, mujeres, adultos mayores y población con destino urbano en México. En estos nuevos grupos de retornados se encuentra el colectivo de interés para el presente artículo: los migrantes de retorno en edad avanzada o adultos mayores, es decir, aquellos de 60 o más años de edad.

Es pertinente profundizar en la experiencia de este tipo de migrantes tras su retorno, ya que proporciona elementos para elaborar políticas públicas focalizadas y contribuye a la visibilización de este colectivo en la bibliografía especializada y la sociedad en general.

Este artículo presenta una reflexión sobre las condiciones en que los migrantes adultos mayores regresan a México, poniendo énfasis en cómo las redes sociales y la familia pueden incentivar su integración laboral y social tras el retorno, así como las características de las desventajas que afrontan. El trabajo se basa en una revisión de la literatura sobre el tema, complementada con el análisis de casos derivados de un trabajo de campo antropológico en dos comunidades del estado de Jalisco.

\section{Breve contexto de la migración de retorno contemporánea de Estados Unidos}

Para entender las condiciones estructurales que se gestaron durante al menos las dos últimas décadas, que tuvieron como resultado un aumento masivo del flujo de retorno de Estados Unidos a México, es importante ubicar este fenómeno como consecuencia de un proceso histórico, político y estructural que no "apareció de pronto" (Gandini et al., 2015). Desde mediados de la década de los noventa, la criminalización de los migrantes indocumentados en Estados Unidos fue persistente, por ejemplo con la Ley de Reconciliación de Responsabilidad Personal y Oportunidad de Trabajo (IIRIRA, por sus siglas en inglés) y la Ley de Reconciliación de Responsabilidad Personal y Oportunidad de Trabajo (PRWORA, por sus siglas en inglés ${ }^{2}$ ), promulgadas en 1996; pero esta perspectiva se intensificó a partir de los

2 Estas leyes han ampliado la posibilidad de que aumenten las detenciones de inmigrantes, así como la restricción del acceso a los servicios de salud para ellos (Ángel, 2013; Jacobo, 2014). 
atentados del 11 de septiembre de 2001, un parteaguas que implicó el endurecimiento constante de la legislación y vigilancia de la población inmigrante en general $^{3}$ en varias esferas del gobierno y toda la geografía estadounidense (Durand \& Massey, 2003; Ángel, 2013).

Por otra parte, la crisis económica y financiera que detonó durante 2008 en Estados Unidos produjo un déficit en la generación de empleos sobre todo en el sector de la construcción, que es uno de los principales nichos de trabajo para los inmigrantes mexicanos (Gandini et al., 2015; Escobar, 2016). La falta de oportunidades laborales en este sector y las repercusiones económicas que tuvo la crisis en años posteriores incentivaron el retorno de mexicanos a su país de origen, con lo que se abrió la posibilidad de continuar con un proyecto de vida para ellos y sus familias en México y se desincentivaron posibles nuevos flujos de emigrantes mexicanos a dicho país. Al mismo tiempo, México ha experimentado un lento pero sólido proceso de crecimiento económico y un rápido cambio demográfico que se ha manifestado en el descenso de las tasas de fecundidad y dependencia, con lo que también disminuye la presión sobre los mercados laborales, que se muestran crecientes. La conjunción de todos estos factores, de distinta índole, ha tenido una clara repercusión en el aumento sustancial del flujo migratorio de retorno a México.

Para dar una imagen general del número de migrantes de retorno, a partir de la crisis de 2008, según estimaciones de BBVA Bancomer y Conapo ${ }^{4}$ (2014) entre 2005 y 2010 se registraron alrededor de 824 mil migrantes de retorno de Estados Unidos a México, mientras que en el quinquenio 2010-2015 retornaron 495,434 (BBVA Bancomer y Conapo, 2017).

Un aspecto que es importante resaltar es que la migración irregular a Estados Unidos, sobre todo hasta la década de los ochenta, era predominantemente circular y por periodos de tiempo, por lo que el retorno era continuo pues no existían las condiciones necesarias para que los inmigrantes se asentaran en aquel país (Riosmena \& Massey, 2012).

Sin embargo, la última gran reforma migratoria en Estados Unidos mediante la Ley de Reforma y Control de la Inmigración (IRCA, ${ }^{5}$ por sus siglas en inglés) de 1986 hizo posible la regularización de los inmigrantes indocumentados e inició un nuevo periodo de asentamientos de migrantes mexicanos y la reagrupación de familias en

En este periodo la persecución contra inmigrantes procedentes de países musulmanes ha sido claramente más agresiva que contra otras poblaciones de inmigrantes en Estados Unidos.

$4 \quad$ Estas estimaciones se realizaron con base en información de los censos de población y vivienda de 2000 y 2010, y de la Encuesta Intercensal 2015.

5 Con la IRCA se regularizó la situación migratoria de 2.3 millones de mexicanos, lo cual ocasionó un nuevo flujo de migrantes a Estados Unidos que permitió la reunificación de familias en aquel país. 
ese país. El retorno de los inmigrantes que pudieron regularizar su situación migratoria pudo sostenerse de manera continua ya que podían moverse con mayor libertad entre los dos países. Sin embargo, para los indocumentados el retorno a México representó cada vez más dificultades y aumentó el costo y riesgo de volver a ingresar en Estados Unidos. Por eso la circularidad migratoria disminuyó o se interrumpió, y esto ocasionó que los inmigrantes irregulares se establecieran por periodos mucho más largos en dicho país (Durand \& Massey, 2003).

El volumen de migrantes mexicanos indocumentados en Estados Unidos alcanzó un pico de 6.9 millones en 2007 —antes de iniciar la crisis económica-, de un total de 12 millones de migrantes mexicanos en dicho año (Passel \& Cohn, 2016).

El perfil del migrante de retorno que se identificaba con el patrón clásico de migración circular entre México y Estados Unidos correspondía predominantemente a varones en edad productiva o que esperaban su etapa de jubilación después de concluir su trayectoria laboral en dicho país y regresaban a esperar la muerte en su lugar de origen. López (1998) señala este tipo de migrante retirado como parte de una serie de prenociones que se tenían en la academia sobre el migrante de retorno. Sin embargo, este perfil "tradicional" del migrante de retorno, ampliamente identificado en la bibliografía académica de la migración, cambió por completo en la última década.

Una de las variaciones en el grupo de migrantes de retorno es la de los adultos mayores que regresan a México en condiciones de precariedad laboral, económica y de salud. A continuación se presenta una discusión acerca de los hallazgos recientes sobre los migrantes de retorno en edad avanzada, la cual permitirá mostrar los contrastes entre los migrantes adultos mayores que consiguieron un estatus migratorio regular que les permitió contar con una pensión - los migrantes "exitosos" - y los que, por diversas circunstancias, retornaron como indocumentados.

\section{Reflexiones en torno a los adultos mayores migrantes de retorno}

En el caso de la migración mexicana, la imagen tradicional de un adulto mayor que es migrante de retorno se apoyaba en ciertas prerrogativas. Por ejemplo, se basaba en la idea de que el retorno es la última etapa en la trayectoria migratoria de las personas y representa un asentamiento permanente y definitivo en la localidad de origen (Gandini et al., 2015). Adicionalmente a esta visión clásica de la migración de retorno, se generó un juicio de valor entre los habitantes de las zonas de origen sobre lo que se consideraba un migrante de retorno exitoso o fracasado (Durand, 2004). El migrante "exitoso" regresa a invertir y estimular el desarrollo económico de su región (Papail, 2002, 2005; Papail \& Arroyo, 2009), el fracasado regresa sin nada. 
Bajo esta premisa, se agrupaba a todas las personas en etapa de jubilación en una sola categoría: aquellos migrantes que concluyeron con éxito su experiencia migratoria. Por ejemplo los que cuentan con un estatus migratorio documentado que les permite regresar a México y recibir algún tipo de beneficio de la seguridad social desde Estados Unidos, o que consiguieron hacer un ahorro, construir una casa, comprar un rancho o ganado, o invertir en un negocio que les apoyaría en su manutención al regreso. Es recurrente encontrar amplia bibliografía que se refiere a estos migrantes en edad avanzada que cumplieron los objetivos de su trayectoria migratoria (Durand, 2004; Mestries, 2013; Mojica, 2016).

Tanto el concepto de migración de retorno como el de migración definitiva al final de la vida productiva y el de la idea del migrante exitoso que regresa a su lugar de origen han sido cuestionados por diversos autores, quienes proponen problematizar estos conceptos porque la evidencia empírica muestra que el proceso de migración de retorno es complejo e incorpora otros escenarios y sujetos migrantes (Rivera, 2011; Moctezuma, 2013; Zenteno, 2012, Woo \& Flores, 2015, Gandini et al., 2015).

En el panorama reciente de la migración de retorno de Estados Unidos a México, si bien se observa que hay un claro aumento de migrantes de retorno en rangos etarios más cercanos a los de jóvenes, niños y niñas ${ }^{6}$ (Gandini et al., 2015), también se presenta un considerable aumento de la población de 60 años y más que regresa al país. Muestra de ello es que entre 2000 y 2010 aumentó el retorno de adultos mayores en $158 \%$, lo que en números absolutos representa alrededor de 35 mil adultos mayores (García \& Gaspar, 2016). Aun cuando no se pueden identificar las causas específicas del retorno, por medio de estimaciones censales se puede encontrar que la reunificación familiar, los problemas de salud o la falta de empleo son algunos de los motivos de retorno de los adultos mayores.

Aunque en general la población mexicana presenta una tendencia al envejecimiento y se prevé que habrá un aumento de adultos mayores en la siguiente década, es importante mencionar que este sector también crece con la presencia de migrantes de retorno en México (Riosmena, González, \& Wong, 2013). Aunado a lo anterior, la ausencia de políticas públicas e instituciones focalizadas en la atención de este tipo de población agrava su situación, ya de por sí precaria, porque se le ofrecen muy pocas posibilidades de acceso a servicios de salud, vivienda y empleo. En este sentido, existen discrepancias entre las evidencias aportadas por los pocos estudios que se han realizado sobre las condiciones de los migrantes de retorno en edad avanzada. Por una parte, algunos sostienen que los adultos mayores que tuvieron experiencia migratoria y regresan a México tienen ventajas económicas y de salud

6

Me refiero en este punto a los casi 600 mil menores de edad nacidos en Estados Unidos que llegaron a México entre 2005 y 2010 con su padre o madre de origen mexicano (INEGI, 2010). 
respecto a la población en la misma edad que no ha sido migrante (Wong, Palloni, \& Soldo, 2007; Riosmena et al., 2013).

También Gualda y Esteva (2012) encuentran que los retornados de edad avanzada tienen una concepción de bienestar distinta de la que tuvieron antes de la experiencia migratoria y privilegian condiciones como los lazos familiares y sociales, que los ayudan a tener mejor calidad de vida en la vejez. Si bien las condiciones materiales pueden ser heterogéneas, la mayoría resaltan la importancia de tener relaciones sociales al regreso y contar con algún tipo de pensión y actividades recreativas, cívicas o de empleo para mantenerse activos. Sin embargo, también existen condiciones de precariedad y desventaja, que se acentúan cuando los migrantes de retorno presentan algún tipo de enfermedad, discapacidad o baja captación de ingresos, además de que tienen una menor cobertura de seguridad social y servicios de salud (Riosmena et al., 2013; Águila y Zissimopoulos, 2013; García \& Gaspar, 2016).

En este sentido, Ullmann, Goldman y Massey (2011) encuentran que los migrantes de retorno varones presentan peor condición de salud en enfermedades del corazón, trastornos psíquicos y emocionales, obesidad y tabaquismo que la población no migrante. Refieren que aun cuando no se le puede atribuir una sola explicación a este hecho, es posible que esté relacionado con el de que la experiencia migratoria provoca una mayor frecuencia de estos padecimientos entre los migrantes, pero también explican que el deterioro de su salud puede ser la razón de su retorno. En todo caso, los hallazgos del estudio sugieren que el estrés asociado a la vida en Estados Unidos puede incrementar la proclividad a padecer psicopatologías tras el retorno. Sin embargo, Ullmann et al. (2011) identifican también que en la evaluación que los propios migrantes de retorno hacen sobre su estado de salud estos padecimientos no parecen tener más importancia que otros aspectos que consideran parte del bienestar, como el estatus socioeconómico o la satisfacción personal, lo cual indicaría que entre los migrantes prevalece una valoración más alta de otros aspectos de su vida cotidiana en el retorno que su propia salud.

Mojica (2016) refuerza el argumento de que los migrantes de retorno en edad avanzada presentan trastornos emocionales y psicológicos a raíz de separaciones familiares derivadas del regreso, o cuando este se hace en solitario. Aunque en algunos casos se han cumplido sus expectativas materiales o económicas, si su familia se queda en Estados Unidos la decisión del retorno no se experimenta como una situación de éxito o satisfactoria.

Así, los estudios mencionados proveen evidencia disímil respecto a las condiciones y experiencias del retorno en adultos mayores, por lo que en la siguiente sección se profundiza con información etnográfica de casos específicos para discutir 
algunos de estos hallazgos e incorporar categorías de análisis que contribuyan a explicar algunos procesos de integración de estos migrantes de retorno y las problemáticas que enfrentan.

\section{Redes sociales, familia y acumulación de desventajas}

En este apartado se presentan los casos de migrantes adultos mayores que retornaron a su lugar de origen después de una larga trayectoria migratoria. A través de redes sociales, familiares y grupos de apoyo, sortean las dificultades diarias de su condición, pero esto no siempre es suficiente para satisfacer las necesidades que tienen, derivadas de su estado de salud o falta de recursos económicos. Su integración social suele ser efectiva, ya que recuperan lazos de amistad y familiares que les permiten sentirse acogidos de nuevo, pero las enfermedades y la edad avanzada hacen complicada la integración laboral y la mínima estabilidad económica que requieren para subsistir. Su estatus migratorio irregular en Estados Unidos y otras experiencias de la trayectoria migratoria han dejado a algunas personas en un estado físico, económico y emocional inestable.

Para fines analíticos, en esta sección utilizo el concepto de acumulación de desventajas, que se refiere a los procesos que se caracterizan por presentar una serie de eventos (desventajas) que hacen más o menos capaces de enfrentar crisis de distinta índole a personas, hogares, comunidades o sociedades (González de la Rocha, 2016) ante una situación contingente.

Los recursos económicos, sociales, laborales y otros con los que cuentan las personas o grupos domésticos se pueden ir deteriorando gradualmente con ciertas condiciones adversas que se presentan en las trayectorias personales o familiares. $\mathrm{Si}$ un recurso se desgasta es común que eventualmente otro también lo haga como consecuencia de este proceso de deterioro de los recursos, y esto hace que se reduzca la capacidad de sobreponerse a crisis o eventos inesperados como enfermedades, pérdida del empleo, accidentes, etc. Las desventajas, entonces, pueden presentarse de múltiples formas e "impide[n] a quienes la sufren, hombres y mujeres, alcanzar oportunidades genuinas para gozar una vida con menos riesgos, más libertad y mayores satisfacciones" (González de la Rocha, 2016, p. 20).

A continuación, a través de dos casos, se ejemplifica de manera más clara la aplicación del concepto de acumulación de desventajas y las condiciones en que los migrantes de retorno adultos mayores regresan a sus lugares de origen. 
Belén y Uri son hermanas, se llevan de diferencia tan solo un año de edad, tienen $66 \mathrm{y}$ 65 años respectivamente y vivieron 24 y 22 años en Estados Unidos. Al morir sus padres, cuando ellas tenían alrededor de cuarenta años, decidieron viajar al norte para trabajar y conseguir una mejor calidad de vida, ninguna de las dos volvió a México en todo ese tiempo pero siempre conservaron la idea de regresar. Uri estuvo casada en México pero su matrimonio fracasó y no tuvo hijos, Belén nunca se casó y tampoco los tuvo.

La edad y el ciclo de vida tanto en la migración como en el retorno pueden marcar una diferencia en la experiencia migratoria. Para un migrante representa una desventaja llegar al lugar de destino a una edad en que le queda poco tiempo de vida productiva, condición que puede mermar sus objetivos de crear un patrimonio material y económico. En el proceso de retorno, es mucho más complicado para los migrantes adultos mayores incorporarse a un empleo, ya que existe una generalizada discriminación laboral hacia la población en estas edades (Riosmena et al., 2013).

Conocí a las hermanas mientras realizaban un trámite para obtener un apoyo gubernamental en especie por el Fondo de Apoyo a Migrantes (FAM), programa dirigido a migrantes de retorno que es hasta ahora el único focalizado en la atención de ciertas necesidades de esta población, pero que ha quedado ampliamente rebasado en su alcance y cobertura (García \& Del Valle, 2016).

Durante su estancia en Estados Unidos trabajaron en diversos oficios, desde la pizca en el campo hasta en fábricas y cuidando adultos mayores. Siempre vivieron juntas y se desplazaron por varios lugares del estado de California. Llegaron solas a Estados Unidos; tenían algunos familiares en ese país pero radicaban lejos de la ciudad donde ellas vivían y no les gustaba la idea de vivir con familiares. Trataron de regularizar su estatus migratorio pero no lo consiguieron, Belén trabajó con un número de seguridad social falso o prestado.

Cuentan que las dos enfermaron de diabetes; la edad y la enfermedad les impedían trabajar ya que comenzaron a perder la vista. Sin embargo, las deudas no conocían de enfermedades y cada mes se les dificultaba más pagar la renta y los demás gastos corrientes a pesar de que en Estados Unidos contaban con apoyos gubernamentales por ser personas mayores de edad. Sin embargo, la atención médica que recibieron en ocasiones les pareció inadecuada e incluso perjudicial para su estado de salud.

La imposibilidad de trabajar y la enfermedad hizo que decidieran regresar a su pueblo. Tiempo después de conocerlas, las visité en su casa en una pequeña localidad del municipio de San Gabriel y constaté la ausencia de transporte público y de vías de comunicación entre esta comunidad y la cabecera municipal. Me comentaron que 
solo podían ir a San Gabriel si algún vecino o familiar las trasladaba en vehículo, ya que para ellas resultaba prácticamente imposible trasladarse por cuenta propia.

En 2014, regresaron a la destruida y abandonada casa de sus padres en el pueblo; al volver se dieron cuenta de que la vivienda había sido asaltada y se habían llevado prácticamente todo lo que había en su interior. Mientras hacían arreglos para poder habitarla, estuvieron viviendo con un familiar.

El hecho de retornar a su lugar de nacimiento y la socialización ha sido para ellas un factor de apoyo en su integración social. Conocen las normas y los valores que predominan en el lugar, así como las dinámicas sociales entre los habitantes, a pesar de haber estado ausentes tanto tiempo.

Sin hijos ni pareja, ni poder trabajar, las hermanas se mantienen gracias a la ayuda de la familia extensa, en particular de sobrinos, conocidos y vecinos del pueblo, que les hacen llegar dinero para el pago de servicios o les dan alimentos, pero esta ayuda no siempre es posible. La pérdida de vínculos familiares en el lugar de origen está asociada a una situación de desventaja, ya que no cuentan con redes de apoyo constantes, lo que les produce inestabilidad y riesgo en su entorno.

Estos casos coinciden con lo que García y Gaspar (2016) encuentran sobre las mujeres en retorno que presentan desventajas en cuanto al acceso a servicios de salud, pensión e ingresos.

A pesar de haber trabajado más de veinte años en Estados Unidos, el estatus irregular de las hermanas en aquel país no les permitió pedir una pensión para pasar su vejez y atender su enfermedad. Si bien reciben apoyo gubernamental en México por ser adultos mayores - a través del programa 65 y más, que les otorga un poco más de mil pesos bimestrales-, este ingreso no es suficiente para cubrir los gastos y satisfacer sus necesidades diarias.

Estas hermanas presentan las siguientes desventajas, que de manera acumulada provocan que tengan poca capacidad de respuesta ante eventualidades económicas y de salud:

- Padecimiento de una enfermedad crónico-degenerativa (diabetes) que les ha provocado la pérdida de la vista.

- Imposibilidad de trabajar debido al avanzado estado de su enfermedad y a su edad, lo que repercute en su nivel de ingresos y recursos económicos.

- Pocas redes de apoyo, pues ya no existe su familia nuclear ni tuvieron familia propia.

- Mala calidad de su vivienda.

- Imposibilidad de trasladarse por cuenta propia a otros lugares fuera de su lugar de residencia, por ejemplo a la cabecera municipal para obtener servicios de salud o ser atendidas por programas sociales. 


\section{"Andrés"}

Sobre una de las principales arterias viales de Guadalajara, la avenida Alcalde, donde el ruido, el tránsito y el movimiento de personas es patente todos los días, está el pequeño y humilde taller mecánico de Andrés. Pasa casi inadvertido, ya que solo es un pedazo de cochera al borde de la banqueta. Hay un auto atravesado en medio del lugar, que ocupa prácticamente todo el espacio del recinto. Andrés me invita a sentarme a un lado del auto, me acerca un banco y él toma otro, así me comienza a narrar su historia.

Andrés era muy joven cuando se fue a Estados Unidos, tenía veinte años. Lo convencieron de que tenía talento para el futbol y allá tendría oportunidades de jugar como profesional. Corría el año de 1968. Como Andrés tenía ganas de conocer dicho país y trabajar allá para ganar dinero con el cual poner una farmacia, no dudo en embarcarse en la aventura. Luego de 49 años, regresó a su ciudad natal, ya con 69 años de edad.

Lo del futbol le duró poco, pero Andrés encontró trabajo pronto como obrero en fábricas de lámparas o de mecánico de camiones de basura, donde aprendió el oficio que hoy le ayuda a sobrevivir. Ya había obtenido la residencia legal en Estados Unidos pero entonces fue cuando tuvo "el problema", como él lo llama. Un día, varios agentes de la policía irrumpieron en su casa sin orden de cateo; le preguntaban si ahí había droga y quiénes eran los vendedores. Andrés había visto algunas cajas y bolsas extrañas en la casa que compartía con otros familiares pero se limitaba a pasar de largo, nunca preguntó ni se enteró de nada. Cuando los agentes le inquirieron si él estaba involucrado, con su cortado y deficiente inglés, expresó que sí había visto cajas en la casa pero no sabía más. Esta declaración fue suficiente para que Andrés pasara ocho años de su vida en una cárcel de Los Ángeles, incriminado por tráfico de drogas, y después fuera deportado a México.

De acuerdo con su testimonio, el proceso estuvo plagado de inconsistencias e injusticias hacia él. Le quitaron su residencia y el derecho que ya le correspondía a recibir una pensión por años trabajados, y además sufrió la separación de su esposa cuando lo sentenciaron. Este par de hechos parecen haber afectado por algún tiempo el proceso de integración de Andrés, sobre todo en lo relativo a su relación familiar y el retiro de su estatus de residente legal en Estados Unidos, que le impidió tener una jubilación por su trayectoria laboral. Al no contar con ingresos, para él fue complicado comenzar con un empleo estable. Aun ahora la estabilidad económica de Andrés es variable y no le deja margen para eventualidades como enfermedades, emergencias o gastos extras.

Pese a todo, se siente contento y satisfecho de lo que logró a partir de su trabajo duro en Estados Unidos; le mandaba dinero a su mamá, hizo una casa donde ahora 
viven sus hijos y su exesposa y aprendió el oficio de mecánico. Esto, simbólicamente, representa un éxito en su trayectoria migratoria, que se expresa en la consecución de un objetivo económico y patrimonial; sin embargo, no representa una ventaja real en el retorno porque no tiene ese bien patrimonial.

En este punto es útil retomar el planteamiento de Ullman et al. (2011) sobre la propensión que presentan los migrantes de retorno varones respecto a sufrir padecimientos psicológicos derivados de su experiencia de vida en Estados Unidos. A Andrés, el hecho de haber estado en prisión, ser deportado, perder su residencia legal en ese país y su separación conyugal lo han afectado de manera importante en el proceso de integración a su lugar de origen; sin embargo, a pesar de ello, de acuerdo con el argumento de Ullman et al. (2011), hace una valoración positiva de su experiencia migratoria porque conseguió dar estudio y patrimonio a su familia, aun cuando él en estos momentos, tras el retorno, no cuente con recursos suficientes para símismo.

El ciclo de vida y familiar de Andrés se relaciona con una etapa en que el retorno al lugar de origen le da un sentido de tranquilidad y sosiego, se añora el lugar de origen por concederle esa idea de descanso en la etapa final de la vida (Espinosa, 1998). Él mismo menciona que valora estar en Guadalajara porque puede disfrutar a sus hermanos y hermanas, salir a pasear o comer con amistades, lo cual no hacía con frecuencia en Estados Unidos.

Cuando llegó a Guadalajara estuvo viviendo en la casa que era de su madre, conservó lazos familiares con sus hermanos y sus amigos, que a su regreso le permitieron conseguir trabajo y tener un espacio propio para vivir y trabajar. Una familia a la que recibía de visita en Estados Unidos ahora le presta la casa donde vive y que adaptó como taller mecánico. En este punto se observa una situación de reciprocidad social, que se refiere a los intercambios entre personas en distintos momentos y motivados por varias razones pero que ayudan a reproducir lazos de solidaridad y apoyo en un círculo social o familiar cuando alguno de sus integrantes enfrenta situaciones contingentes o de emergencia (González, 1999). Esta reciprocidad se puede considerar como un elemento de integración.

El hecho de haber nacido en Guadalajara y tener aún lazos afectivos con distintas personas le han ayudado a establecerse de nuevo. Tiene varios hermanos que viven en la misma ciudad, además de hijos e hijas.

Aunque Andrés no padece ninguna enfermedad crónica —al menos eso cree-, tampoco tiene atención médica periódica ni seguridad social. Lo que gana en su modesto taller lo usa para mantenerse ya que le prestan la casa donde vive. Le han dicho del programa de 65 y más pero no sabe dónde solicitarlo y le representa perder un día de trabajo ir a preguntar. 
En el caso de Andrés se presenta la siguiente acumulación de desventajas:

- Falta de patrimonio propio, en particular de una casa donde vivir.

- Ingresos económicos que resultarían insuficientes para una eventual enfermedad o emergencia.

- Experiencia de un trato legal injusto en Estados Unidos, que no le ha permitido recibir la pensión que le corresponde por su trayectoria laboral en ese país.

- Experiencia de separación conyugal antes del retorno.

A partir de estas experiencias, se pueden observar algunos factores que contribuyen a la integración laboral y social de los migrantes de retorno y otros que les obstaculizan la vida tras el regreso. En la siguiente sección se presenta una discusión sobre estos casos en relación con la literatura revisada para resaltar las variaciones o coincidencias que existen entre la evidencia empírica de los migrantes de retorno que son adultos mayores.

\section{Discusión}

Se puede pensar que las desventajas de los casos expuestos están poco asociadas a la experiencia migratoria ya que el deterioro en la salud, la falta de ingresos o de patrimonio es una condición general del grueso de la población mexicana en edad avanzada. Sin embargo, esta discusión se propone distinguir las desventajas que pueden ligarse a la experiencia migratoria y se experimentan tras el retorno a México.

Una variedad de estudios (Viruell Fuentes, Miranda, \& Abdulrahim, 2012; Viruell Fuentes, 2007) han profundizado en la influencia que ejercen ciertas condiciones estructurales en Estados Unidos, a las cuales los inmigrantes ${ }^{7}$ están expuestos y afectan la calidad de su salud física y emocional. Entre estas condiciones se mencionan el estrés asociado al racismo, la segregación espacial, la falta de acceso a servicios de salud, los factores ambientales y de riesgo, etc. A partir de estas investigaciones, se establece un vínculo entre este tipo de situaciones que se viven en la experiencia migratoria y el deterioro de la salud de los inmigrantes.

Anteriormente se mencionó que también Ullmann et al. (2011) encuentran que algunas enfermedades que se presentan en los migrantes de retorno se pueden relacionar con el estrés que provoca el ritmo de trabajo y de vida que llevaron en Estados Unidos. Lo que es importante destacar de estas propuestas es que se visibilizan una serie de condiciones estructurales presentes en dicho país y que intervienen como factores negativos en la salud de los inmigrantes. Esta premisa permite

7 Estos estudios analizan a varios colectivos de inmigrantes en Estados Unidos, entre ellos los mexicanos. 
reflexionar que existe relación entre las afectaciones físicas y emocionales de los migrantes de retorno y su experiencia migratoria, por lo que podría ser factible observar la diferencia entre los procesos de enfermedad de los migrantes de retorno originados a raíz de la migración y los de la población no migrante.

Es importante destacar que un aporte que hacen los testimonios de estos migrantes es visibilizar las problemáticas específicas de la integración de adultos mayores en retorno, pues se cuestiona la idea de que todos los migrantes en edad avanzada son heterogéneos; que regresan después de una experiencia migratoria exitosa, sin problemas de salud y que su retorno está exento de contradicciones y dificultades familiares y sociales.

El deterioro en la salud de las hermanas se generó y agravó mientras estuvieron en Estados Unidos, ellas perciben que hubo procedimientos y servicios médicos que empeoraron su situación física. En este sentido, puede relacionarse que la falta de acceso a los servicios de salud en ese país y su calidad tuvo consecuencias negativas en la salud de las hermanas. También, el hecho de haber mantenido un estatus migratorio irregular les impidió obtener una pensión después de los años de trabajo productivo, lo cual tras el retorno es una franca desventaja en la generación de recursos económicos.

En lo que respecta a las desventajas económicas, patrimoniales y familiares que acumulan los protagonistas de los dos casos revisados, también se puede identificar un componente relacionado con la experiencia migratoria. Andrés, por ejemplo, perdió el derecho a la pensión por jubilación después de su proceso legal en Estados Unidos, lo que le ocasionó inestabilidad económica, además de la separación de su cónyuge, perdiendo así un lazo emocional y afectivo importante.

Por su parte, las hermanas experimentaron la pérdida de relaciones familiares y personales durante el largo tiempo que pasaron en Estados Unidos. Aunque han podido recuperar las redes de apoyo con la familia extensa y los vecinos, estos apoyos se ven seriamente comprometidos ante una situación más crítica de crisis económica o de salud.

De acuerdo con Riosmena et al. (2013), la derechohabiencia a servicios de salud de los migrantes de retorno que son adultos mayores es menor respecto a la que tiene la población no migrante, así como la oportunidad de insertarse en el mercado laboral formal que les permita recibir beneficios sociales. Los dos casos presentados proporcionan evidencia que refuerza este argumento, pues ninguno de estos migrantes de retorno ha podido conseguir un empleo formal ni asegurar la atención médica, y existen otras desventajas que se suman a su condición. 


\section{Reflexiones finales}

La presencia o ausencia de redes sociales y familiares quizá sea uno de los factores que más inciden en el proceso de integración de los migrantes de retorno. Es a partir de las relaciones sociales que los migrantes conservaron -o reactivan al regresocomo pueden sortear ciertos obstáculos que se presentan en el asentamiento en los contextos de recepción tras el retorno. En el caso de los adultos mayores estas redes desempeñan un papel fundamental en su estabilidad e integración, ya que proveen ciertos apoyos que permiten solventar algunas desventajas derivadas de su experiencia migratoria y de la precaria situación en la que se encuentran al retornar. Sin embargo, se enfrentan a una constante situación de riesgo y escasa capacidad de respuesta ante escenarios cambiantes.

Es importante visibilizar los diferentes perfiles que existen entre los migrantes de retorno, así como sus propias problemáticas y procesos de integración. De igual manera, es necesario que en el análisis de los migrantes de retorno en general - y de adultos mayores en particular- se haga evidente la influencia que ejerce la experiencia migratoria en el deterioro de su salud para así dilucidar la relación entre la migración, el retorno y las condiciones físicas y psicológicas de estos migrantes.

Para los migrantes de retorno que son adultos mayores es más difícil integrarse a la esfera laboral formal y obtener servicios de salud de calidad que para la población no migrante (Riosmena et al., 2013), por lo que están ante una serie de desventajas distintas de las que se pueden presentar en los adultos mayores sin experiencia migratoria, además de las otras problemáticas familiares y sociales que ya se mencionaron.

En lo que respecta a la existencia de políticas públicas, son insuficientes y no toman en cuenta las especificidades de los migrantes de retorno. Incluso ellos desconocen muchas de las políticas públicas ya que después de una larga estancia en Estados Unidos no están familiarizados con los programas o las instituciones que atienden a los adultos mayores.

Es un reto para el gobierno mexicano la elaboración de políticas públicas en los tres niveles de gobierno que atiendan a los migrantes adultos mayores en retorno porque estos casos pueden presentarse con cada vez mayor frecuencia, sobre todo si tenemos en cuenta que la etapa de migración acelerada a Estados Unidos se llevó a cabo en la década de los noventa. Siguiendo los ciclos de vida de estos migrantes, nos damos cuenta de que ahora tienen o tendrán pronto una edad avanzada y eventualmente pueden regresar a México en condiciones de poca estabilidad económica y física.

En México no hay seguimiento institucional de casos en que los migrantes tendrían derecho legal a obtener un beneficio económico de Estados Unidos 
después de haber cumplido con sus obligaciones laborales, pagado impuestos y contribuido con otros recursos a ese país. Sería adecuado y pertinente que a partir de evidencias como las de estos migrantes en edad avanzada, en situación económica y con salud precaria, se llevara a cabo un lobby político para proponer políticas públicas entre México y Estados Unidos que protejan los derechos laborales de estos migrantes y así puedan obtener la retribución por su trabajo.

También es necesario evidenciar las situaciones de discriminación en el plano institucional que padecen las personas que integran, dirigen o manejan las instituciones públicas en calidad de funcionarios y que excluyen u obstaculizan el ejercicio de derechos sociales a los migrantes de retorno, ya sea en forma directa o indirecta, al darles un trato diferenciado negativo, omitiendo información oportuna y opciones para resolver sus problemáticas. Escobar (2013) encuentra que a migrantes de edad avanzada algunos funcionarios públicos les niegan el programa social de 65 y más, que consiste en la entrega de una módica cantidad de dinero cada bimestre, ya que se tiene el prejuicio de que "no lo merecen" o de que no lo necesitan porque ya "traen pensión de Estados Unidos”.

Tal como se mencionó en otra sección de este artículo, es una realidad que la tendencia al envejecimiento de la población mexicana en la próxima década requerirá de ajustes importantes en la política pública del país, por lo que es necesario tomar en cuenta a la población migrante de retorno en forma transversal en los cambios y las transformaciones de estas políticas.

La reciprocidad social, las redes de apoyo y eventualmente la focalización de políticas públicas para los migrantes de retorno ofrecen posibilidades de integración social y laboral para esta población, por lo que resulta necesario evidenciar las dificultades y desventajas que enfrentan tras el retorno, con el afán de comprender mejor la realidad que viven, y sobre todo emprender acciones concretas desde las instituciones gubernamentales para la atención de sus necesidades.

\section{Referencias}

Águila, E. \& Zissimopoulos, J. (2013). Retirement and health benefits for Mexican migrant workers returning from the United States. International Social Security Review, 66(2), 101-125. Recuperado de http://doi.org/10.1111/issr.12014 [consultado el 29 de noviembre de 2017].

Alanís, F. (2015). Voces de la repatriación. La sociedad mexicana y la repatriación de mexicanos de Estados Unidos 1930-1933. San Luis Potosí: El Colegio de la Frontera Norte, El Colegio de Michoacán. 
Ángel, H. (2013). ¿Un sueño posible? Retos y dificultades que enfrentan los estudiantes mexicanos indocumentados por ingresar a la universidad en Estados Unidos y en México a principios del siglo XXI. Tesis de doctorado, CIESAS-Occidente. Guadalajara, México.

BBVA Bancomer \& Consejo Nacional de Población (Conapo) (2014). Anuario de migración y remesas. México 2014. México: BBVA Bancomer, Conapo.

BBVA Bancomer \& Consejo Nacional de Población (Conapo) (2017). Anuario de migración y remesas. México 2017. México: BBVA Bancomer, Conapo.

Canales, A. (2012). La migración mexicana frente a la crisis económica actual. Crónica de un retorno moderado. Revista Interdisciplinaria, XX(39), 117-134. Recuperado de http://www.scielo.br/pdf/remhu/v20n39/v20n39a07.pdf [consultado el 29 de noviembre de 2017].

Dumont, J. \& Spielvogel, G. (2008). Return migration: a new perspective. En International Migration Outlook (pp. 161-222). París: OCDE. Recuperado de http://www.oecd.org/els/mig/43999382.pdf [consultado el 29 de noviembre de 2017].

Durand, J. (2004). Ensayo teórico sobre la migración de retorno: el principio del rendimiento decreciente. En Delgado, R. \& Kneer, B. (coords.), Contribuciones al análisis de la migración internacional y el desarrollo regional en México (pp. 209318). México: Universidad Autónoma de Zacatecas, Miguel Ángel Porrúa.

Durand, J. \& Massey, D. (2003). Clandestinos: migración México-Estados Unidos en los albores del siglo XXI. México: Miguel Ángel Porrúa.

Durand, J. \& Massey, D. (2003). Clandestinos: migración México-Estados Unidos en los albores del siglo XXI. México: Miguel Ángel Porrúa.

Durand, J. (2013). Nueva fase migratoria. Papeles de Población, 19(77), 83-113. Recuperado de http://www.redalyc.org/pdf/112/11228794004.pdf [consultado el 29 de noviembre de 2017].

Escobar, A. (2013). Migración, migración de retorno y acceso a programas sociales. En Escobar, A., Lowell, L., \& Martin, S. (coords.), Diálogo binacional sobre migrantes mexicanos en Estados Unidos y México. México: CIESAS, Georgetown University.

Escobar, A. (2016). Mexican policy and return migration. En Romo, H. \& Mogollon, O. (eds.), Mexican migration to United States. USA: University of Texas.

Espinosa, V. (1998). El dilema del retorno. Migración, género y permanencia en un contexto transnacional. México: El Colegio de Jalisco, El Colegio de Michoacán.

Freidenberg, J. \& Durand, J. (2016). ¿Cómo hablamos de la migración? Voces de los Estados Unidos y de México: una introducción. Practicing Anthropology, 38(1), 36. doi: http://dx.doi.org/10.17730/0888-4552-38.1.3 [consultado el 29 de noviembre de 2017]. 
Gandini, L., Lozano, F., \& Gaspar, S. (2015). El retorno en el nuevo escenario de la migración entre México y Estados Unidos. México: Consejo Nacional de Población.

García, R. \& Gaspar, S. (2016). Adultos mayores nacidos y residentes en México con vínculos migratorios internacionales (2000-2010). Odisea. Revista de Estudios Migratorios, 3, 151-180.

Giorguli, S., Angoa, M., \& Villaseñor, R. (2014). Los retos ante el nuevo escenario migratorio entre México y Estados Unidos: patrones regionales y políticas locales. En Giorguli, S. \& Ugalde, V. (coords.), Gobierno, territorio y población: las políticas públicas en la mira. México: El Colegio de México.

González, M. (1999). La reciprocidad amenazada: un costo más de la pobreza urbana. En Enríquez, R. (coord.), Hogar, pobreza y bienestar en México. Guadalajara: Instituto Tecnológico y de Estudios Superiores de Occidente.

González Barrera, A. \& Krogstad, J. (2015). What we know about illegal immigration from Mexico. Washington: Pew Research Center.

González de la Rocha, M. (2016). Acumulación de desventajas y vulnerabilidad. En González de la Rocha, M. \& Saraví G. (comps.), Pobreza y vulnerabilidad: debates contemporáneos y desafíos pendientes. En prensa.

Gualda, E. \& Esteva, A. (2012). Diversity in return migration and its impact on old age: the expectations and experiences of returnees in Huelva (Spain). International Migration, 52(5), 178-190.

Instituto Nacional de Estadística y Geografía (INEGI) (2010). Censo Nacional de Población y Vivienda 2010. Aguascalientes: INEGI.

Jacobo, M. (2014). De ida y de vuelta: el impacto de la política migratoria estadounidense en México y su población retornada. Carta Económica Regional, $114,66-91$.

López, G. (1998). Comentario de la contraportada. En Espinosa, V. (1998), El dilema del retorno. Migración, género y permanencia en un contexto transnacional. México: El Colegio de Jalisco, El Colegio de Michoacán.

Massey, D., Pren K., \& Durand, J. (2009). Nuevos escenarios de la migración MéxicoEstados Unidos. Las consecuencias de la guerra antiinmigrante. Papeles de Población, 15(61), 101-128. Recuperado de http://www.reaalyc.org/ articulo.oa?id=11211806006 [consultado el 29 de noviembre de 2017].

Mestries, F. (2013). Los migrantes de retorno ante un futuro incierto. Sociológica, 28(78), 171-212. Recuperado de http://www.scielo.org.mx/scielo.php?script =sci_arttext\&pid=S0187-01732013000100006\&lng=es\&tlng=es [consultado el 26 de marzo de 2014]. 
Moctezuma, M. (2013). Retorno de migrantes a México: su reformulación conceptual. Papeles de Población, 19(77), 149-175. Recuperado de 2014, de http:// www.scielo.org.mx/scielo.php?script $=$ sci_arttext\&pid $=S 1405742520$ 13000300009\&lng=es\&tlng=es. (consultado el 10 de abril de 2014)

Mojica, O. (2016). Retornos sin familia: el caso de migrantes jubilados. Culturales, $I V(2), 79-101$.

Papail, J. (2002). De asalariado a empresario: la reinserción laboral de los migrantes interna-cionales en la región centro-occidente de México. Migraciones Internacionales, 1(3), 79-102.

Papail, J. (2005). Remesas e inversiones de los migrantes de retorno en el centrooccidente de México. En Delgado, R. \& Knerr, B. (coords.), Contribuciones al análisis de la migración internacional y el desarrollo regional en México. México: Universidad Autónoma de Zacatecas, Miguel Ángel Porrúa.

Papail, J. \& Arroyo, J. (2009). Migración a Estados Unidos y autoempleo. Guadalajara, Los Ángeles, México: Universidad de Guadalajara, UCLA Program on Mexico, Profmex World, Juan Pablos Editor.

Passel, J. \& Cohn, D. (2016, September). Overall number of U.S. unauthorized immigrants holds steady since 2009. Pew Research Center.

Passel, J., Cohn, D., \& González Barrera, A. (2012). Net migration from Mexico falls to zero - and perhaps less. Washington D.C.: Pew Hispanic Center.

Riosmena, F. \& Douglas, M. (2012). Pathways to El Norte: origins, destinations, and characteristics of Mexican migrants to the United States. The International Migration Review, 46(1), 3-36.

Riosmena, F., González, C., \& Wong, R. (2013). El retorno reciente de Estados Unidos: salud, bienestar y vulnerabilidad de los adultos mayores. Coyuntura Demográfica, 2, 63-97.

Rivera Sánchez, L. (2011). ¿Quiénes son los retornados? Apuntes sobre el migrante retornado en el México contemporáneo. En Feldman Bianco, B., Rivera Sánchez, L., Stefoni, C., \& Villa Martínez, M. I. (comps.), La construcción social del sujeto migrante en América Latina. Prácticas, representaciones y categorías. Quito: CLACSO, FLACSO, Universidad Alberto Hurtado. Recuperado de http://www. flacsoandes.edu.ec/libros/digital/39541.pdf [consultado el 29 de noviembre de 2017].

Secretaría de Gobernación-Unidad de Política Migratoria (2017). Boletín de Estadísticas Migratorias. Años 2009-2016.

Ullmann, S. H., Goldman, N., \& Massey, D. S. (2011). Healthier before they migrate, less healthy when they return? The health of returned migrants in Mexico. Social Science \& Medicine, 73(3), 421-428. Recuperado de http://www. 
sciencedirect.com/science/article/pii/S027795361100325X?via\%3Dihub [consultado el 29 de noviembre de 2017].

Viruell Fuentes, E. A. (2007). Beyond acculturation: immigration, discrimination, and health research among Mexicans in the United States. Social Science \& Medicine, 65, 1524-1535.

Viruell Fuentes, E. A., Miranda, P., \& Abdulrahim, S. (2012). More than culture: structural racism, intersectionality theory, and immigrant health. Social Science \& Medicine, 75, 2099-2106.

Wong, R., Palloni, A., \& Soldo, B. (2007). Wealth in middle and old age in Mexico: the role of international migration. International Migration Review, 41(1), 127-151.

Woo, O. \& Flores, A. (2015). La migración de retorno de migrantes mexicanos en el siglo XXI. Población y Desarrollo: Argonautas y Caminantes, 11, 23-36.

Woo, O. \& Ortiz, M. (2015). La diversidad de la migración de retorno en Jalisco, estado de la tradición migratoria. Migración y violencia: dos caras del dolor social. En Vázquez Vázquez, D. (coord.), Tlaxcala: Conacyt, El Colegio de Tlaxcala.

Zenteno, R. (2012). Saldo migratorio nulo: el retorno y la política anti-inmigrante. Coyuntura Demográfica, 2, 17-21. 